\title{
(2) OPEN ACCESS \\ Corneal shape changes of the total and posterior cornea after temporal versus nasal clear corneal incision cataract surgery
}

\author{
Ken Hayashi, Tatsuhiko Sato, Motoaki Yoshida, Koichi Yoshimura
}

\begin{abstract}
- Additional material is published online only. To view please visit the journal online (http://dx.doi.org/10.1136/ bjophthalmol-2017-311710).

Hayashi Eye Hospital, Fukuoka, Japan

Correspondence to

Dr Ken Hayashi, Hayashi Eye Hospital, Fukuoka 812-0011, Japan;

hayashi-ken@hayashi.or.jp
\end{abstract}

Received 13 December 2017 Accepted 3 April 2018

Check for updates

To cite: Hayashi K, Sato T,

Yoshida M, et al.

Br J Ophthalmol

2019:103:181-185.

\section{ABSTRACT}

Aim To compare changes in the shape and astigmatism of the total and posterior cornea between eyes with a nasal clear corneal incision $(\mathrm{CCl})$ and eyes with a temporal $\mathrm{CCl}$ in cataract surgery.

Methods The left eyes of 100 patients undergoing phacoemulsification were randomly assigned to undergo a $2.4 \mathrm{~mm}$ nasal (nasal $\mathrm{CCl}$ group) or temporal $\mathrm{CCl}$ (temporal $\mathrm{CCl}$ group). Corneal astigmatic changes were compared between groups using power vector analysis, and corneal shape changes were compared using videokeratography maps at 2 days, and at 2, 4 and 8 weeks postoperatively.

Results The mean $J_{0}$ and $J_{45}$ values of the total cornea were significantly greater in the nasal $\mathrm{CCl}$ group than in the temporal $\mathrm{CCl}$ group at 2 and 4 weeks postoperatively ( $p \leq 0.0392)$, but did not differ significantly between groups at 2 days and 8 weeks. The videokeratography maps revealed a wedge-shaped flattening in the total cornea and a steepening in the posterior cornea around the CCls in both groups at 2 days postoperatively; those were more prominent in the nasal $\mathrm{CCl}$ group than in the temporal $\mathrm{CCl}$ group. The wound-related changes diminished by 8 weeks postoperatively in the total and posterior cornea, and were not markedly different between groups at 8 weeks postoperatively.

Conclusion Corneal shape and astigmatic changes were comparable between groups at 8 weeks postoperatively, although the changes were greater after nasal $\mathrm{CCl}$ than after temporal $\mathrm{CCl}$ in the early postoperative periods, indicating that nasal or temporal $\mathrm{CCl}$ can be selected based on the surgeon's preference.

\section{INTRODUCTION}

Horizontal clear corneal incision (CCI) is the most preferred incision technique in modern cataract surgery ${ }^{1-4}$ Right-handed surgeons make the $\mathrm{CCI}$ on the temporal side in the right eyes. When performing cataract surgery in the left eyes, however, some right-handed surgeons sit at the patient's head to make the CCI on the nasal side, while others sit on the left side of the patient to make the CCI on the temporal side. For surgeons, changing the sitting position when operating on both the left and right sides of the patient may be challenging, or sometimes impossible.

Previous studies demonstrated that surgically induced astigmatism (SIA) is significantly greater after nasal incision than after temporal incision when the incision width is approximately $2.8 \mathrm{~mm}$ or greater. ${ }^{5-9}$ Based on the assumption that the refractive power of the posterior cornea is proportional to that of the anterior cornea, most studies assessed only the changes of the anterior cornea. Recent studies, however, revealed that the posterior corneal astigmatism is not necessarily proportional to the anterior corneal astigmatism. ${ }^{10-13}$ Accordingly, it is necessary to measure the total corneal astigmatism to compare the SIA between various types of incisions. Furthermore, the standard width of CCI has now decreased to $2.4 \mathrm{~mm}$ or less. It is currently unclear whether the shape and astigmatic changes of the total cornea differ between a nasal CCI and temporal CCI with an incision width of $2.4 \mathrm{~mm}$ or less. ${ }^{4}$

The aim of this study was to compare the changes in shape and astigmatism of the total and posterior cornea between eyes that underwent a $2.4 \mathrm{~mm}$ nasal CCI and eyes that underwent a temporal CCI.

\section{PATIENTS AND METHODS}

\section{Study design}

This study was a prospective randomised clinical trial and an exploratory study to compare changes in shape and astigmatism of the total cornea between nasal and temporal CCI. ${ }^{14}$ The study adhered to the tenets of the Declaration of Helsinki. The study was registered at the University Hospital Medical Information Network (UMIN000028385).

\section{Patient recruitment}

A clinical research coordinator screened all consecutive patients scheduled to undergo cataract surgery at the Hayashi Eye Hospital beginning on 17 November 2016. The major inclusion criterion was left eyes scheduled for phacoemulsification with implantation of a single-piece acrylic intraocular lens (IOL; SN60WF, Alcon Laboratories, Fort Worth, Texas, USA). The exclusion criteria were eyes with any pathology of the cornea, optic nerve or macula; eyes with a lens nucleus harder than grade $4^{15}$; eyes with poor mydriasis $(<4.5 \mathrm{~mm})$; eyes with a possible zonular dehiscence or pseudoexfoliation; and eyes with a history of surgery or inflammation. After explanation of the protocol, the patients who met the criteria were asked whether they wished to participate. Written informed consent was obtained from all patients who agreed to participate. Patient enrolment ended on 28 July 2017.

\section{Randomisation}

The patients were randomly assigned to one of two groups. One group comprised patients scheduled 
Table 1 Baseline characteristics and surgical outcomes of the patients with eyes that underwent a $2.4 \mathrm{~mm}$ nasal $\mathrm{CCl}$ (nasal $\mathrm{CCl}$ group) and eyes that underwent a $2.4 \mathrm{~mm}$ temporal $\mathrm{CCl}$ (temporal CCl group)

\begin{tabular}{llll|}
\hline & Nasal CCI group & Temporal CCI group & P values \\
\hline Age (years) & $68.4 \pm 6.4$ & $67.2 \pm 6.6$ & 0.3719 \\
\hline Sex (men/women) & $22 / 28$ & $23 / 27$ & 0.8407 \\
\hline Corneal astigmatism (D) & $0.55 \pm 0.45$ & $0.58 \pm 0.36$ & 0.6674 \\
\hline MRSE (D) & $-2.39 \pm 3.75$ & $-2.18 \pm 4.66$ & 0.8124 \\
\hline Corrected logMAR visual acuity & $0.35 \pm 0.26$ & $0.35 \pm 0.26$ & 0.9726 \\
\hline Pupillary diameter (mm) & $3.60 \pm 0.54$ & $3.57 \pm 0.52$ & 0.7777 \\
\hline Nuclear opalescence & $1.98 \pm 0.35$ & $2.08 \pm 0.29$ & 0.1237 \\
\hline Surgery time (min) & $8.54 \pm 1.83$ & $8.70 \pm 1.30$ & 0.6154 \\
\hline CDE (millijoules) & $8.98 \pm 3.71$ & $8.21 \pm 3.99$ & 0.3199 \\
\hline Irrigation volume (mL) & $56.4 \pm 14.2$ & $54.8 \pm 12.0$ & 0.5444 \\
\hline
\end{tabular}

${ }^{*}$ Corneal astigmatism of the total cornea.

$\mathrm{CCl}$, clear corneal incision; $\mathrm{CDE}$, cumulative dissipated energy; D, dioptre; logMAR, logarithm of minimal angle of resolution; MRSE, manifest spherical equivalent value.

to undergo nasal CCI (nasal CCI group), and the other group comprised patients scheduled to undergo temporal CCI (temporal CCI group). The coordinator generated a randomisation code with equal numbers (1:1 ratio) using computer software and assigned all patients to either the nasal or temporal CCI group on the day before surgery. The coordinator informed the nurse in charge of the surgery room the group to which each patient was assigned several hours before surgery. Just before surgery, the nurse informed the surgeon of the group to which the patient was assigned. The coordinator concealed the assignment schedule until all examinations were completed.

\section{Surgical techniques}

One surgeon $(\mathrm{KH})$ performed all surgeries using surgical techniques, as described previously. ${ }^{16}$ First, two side ports were made $90^{\circ}$ away from the CCI. Next, the surgeon created a continuous curvilinear capsulorrhexis of $\sim 5.0 \mathrm{~mm}$ using a needle. A $2.4 \mathrm{~mm}$ single-plane CCI was made from the limbus using a keratome at the 9 o'clock meridian in the nasal CCI group and at the 3 o'clock meridian in the temporal CCI group. The surgeon estimated the incision meridian and made the CCI $\sim 1.5 \mathrm{~mm}$ in length. After hydrodissection, nuclear phacoemulsification and cortical aspiration were conducted. The anterior chamber was filled with viscoelastic material, and the IOL was placed in the capsular bag using the Monarch II injector with a D cartridge (Alcon). On aspirating the viscoelastic material, all wounds were

Table 2 Comparison of the mean $( \pm S D)$ magnitude of surgically induced astigmatism of the total cornea between eyes that underwent a $2.4 \mathrm{~mm}$ nasal clear corneal incision (CCl; nasal $\mathrm{CCl}$ group) and eyes that underwent a $2.4 \mathrm{~mm}$ temporal $\mathrm{CCl}$ (temporal $\mathrm{CCl}$ group)

\begin{tabular}{llll}
\hline & Nasal CCI group & $\begin{array}{l}\text { Temporal CCI } \\
\text { group }\end{array}$ & P values \\
\hline 2 days postoperatively & $0.88 \pm 0.56$ & $0.63 \pm 0.44$ & $0.0151^{*}$ \\
\hline 2 weeks postoperatively & $0.71 \pm 0.39$ & $0.54 \pm 0.35$ & $0.0214^{*}$ \\
\hline 4 weeks postoperatively & $0.63 \pm 0.36$ & $0.48 \pm 0.35$ & 0.0352 \\
\hline 8 weeks postoperatively & $0.58 \pm 0.39$ & $0.45 \pm 0.42$ & 0.1098 \\
\hline * Statistically significant difference between groups. & \\
\hline
\end{tabular}

thoroughly hydrated and closed with balanced saline solution without sutures.

\section{Astigmatism analysis}

All patients underwent examinations by videokeratography (Topographic Modeling System V.5 (TMS-5), Tomey, Nagoya, Japan) preoperatively, and at 2 days, and at 2, 4 and 8 weeks postoperatively. The TMS-5 includes a rotating Scheimpflug system and Placido-ring topographer. This device obtains topographic data of the total, anterior and posterior cornea by merging Placido-ring topography with the Scheimpflug system. The magnitude and meridian of the corneal astigmatism of the total, anterior and posterior cornea were determined with the simulated keratometric $(\mathrm{K})$ values. The reproducibility of the $\mathrm{K}$ values was confirmed in previous studies. ${ }^{17-19}$ SIA was calculated as the change from the preoperative astigmatism to the postoperative astigmatism at each postoperative interval. The SIA was decomposed into vertical $\left(90^{\circ}\right)$ /horizontal $\left(180^{\circ}\right)$ astigmatic changes and oblique changes $\left(45^{\circ}\right.$ and $\left.135^{\circ}\right)$ using power vector analysis. ${ }^{20}$ This analysis expresses the vertical/horizontal change as $\mathrm{J}_{0}$, and the oblique change as $\mathrm{J}_{45}$. In the total cornea, a positive $\mathrm{J}_{0}$ indicates a with-the-rule astigmatic change, while a negative $\mathrm{J}_{0}$ indicates an against-the-rule change. In the posterior cornea, a positive $\mathrm{J}_{0}$ indicates against-the-rule astigmatic change, while a negative $\mathrm{J}_{0}$ indicates with-the-rule change. In the total cornea, a positive $\mathrm{J}_{45}$ indicates a clockwise oblique astigmatic change, while a negative $\mathrm{J}_{45}$ indicates a counter-clockwise oblique change.

Irregular astigmatism was calculated by Fourier harmonic transformation, which was described previously. ${ }^{416}$ Briefly, the refractive powers of the total cornea are transformed into four trigonometric components: the spherical equivalent power, the first-order asymmetry, the second-order regular astigmatism, and the third and higher order irregularity. The asymmetry and higher order irregularity are defined as irregular astigmatism. The calculations were performed for a $6.0 \mathrm{~mm}$ central optical zone.

\section{Evaluation of surgically induced changes in corneal shape}

The mean changes in the shape of the total and posterior cornea were assessed using the average of difference map of the TMS-5. The mean corneal shape changes were determined as the change from the preoperative shape to the postoperative shape at each postoperative interval. The average of difference map was produced by subtracting the mean postoperative data from the preoperative data with the mean corneal diameter of approximately $8.9 \mathrm{~mm}$. This map displays between -5.0 dioptre (D) and $7.5 \mathrm{D}$ in $0.5 \mathrm{D}$ steps.

\section{Secondary outcome measures}

Uncorrected (UDVA) and corrected distance visual acuity (CDVA) were examined on decimal charts, and these acuities were converted to the logarithm of minimal angle of resolution scale for statistical analysis. The length and meridian of CCIs were examined at 2 weeks postoperatively using an anterior segment-optical coherence tomography (SS-1000; Tomey). The ocular higher order aberrations (HOAs) were measured using a Hartmann-Shack wavefront aberrometer (KR-1W: Topcon, Tokyo, Japan). ${ }^{21}$ Ocular HOAs were measured in the central 6.0 mm optical zone.

\section{Statistical analysis}

The normality of the data distribution was examined by inspecting histograms. The magnitude of the SIA, $\mathrm{J}_{0}$ and $\mathrm{J}_{45}$, 


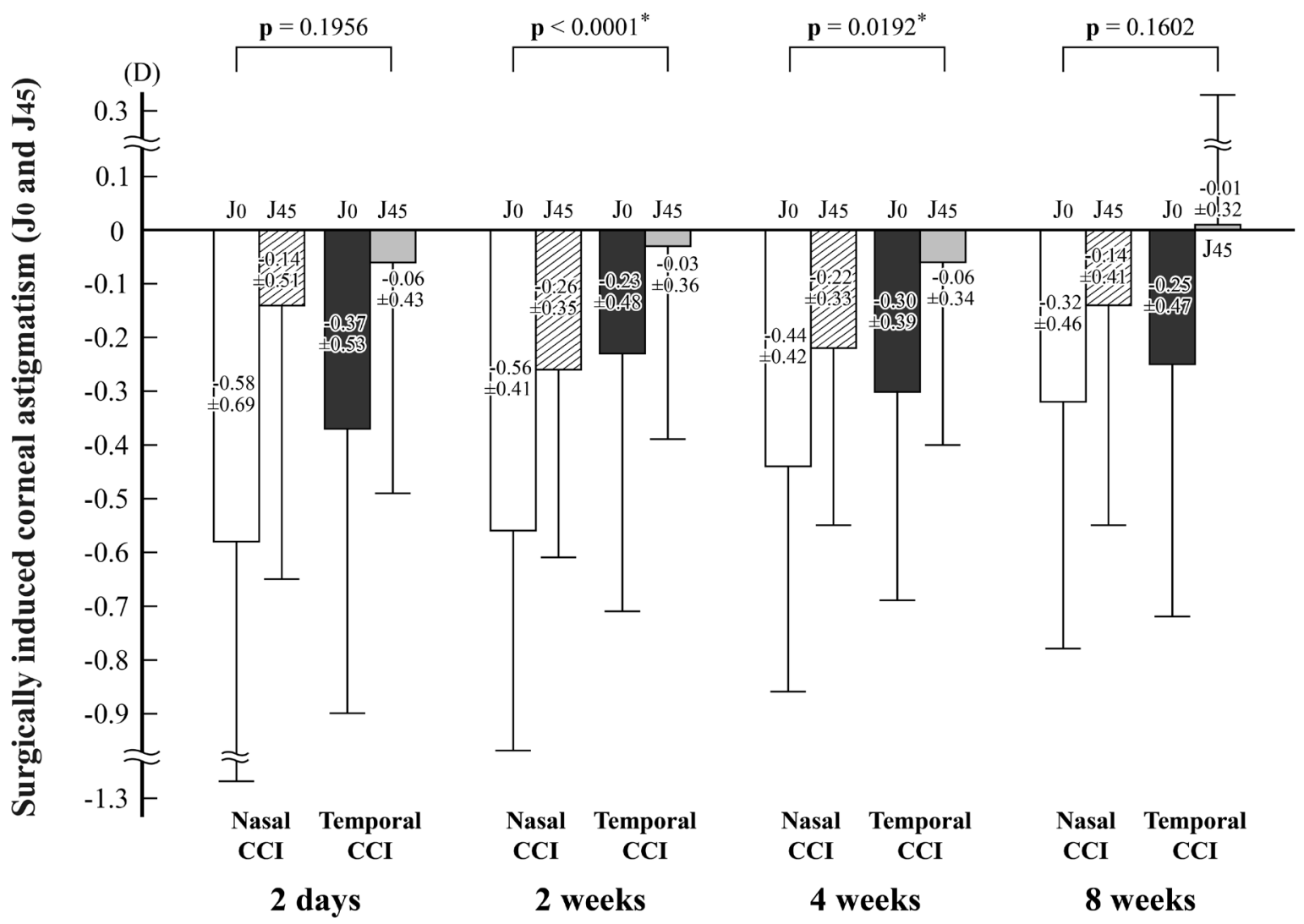

Figure 1 Multivariate comparison of the mean $( \pm S D)$ astigmatic changes $\left(J_{0}\right.$ and $\left.J_{45}\right)$ of the total cornea calculated using power vector analysis between eyes that underwent a $2.4 \mathrm{~mm}$ nasal clear corneal incision (CCl; nasal $\mathrm{CCl}$ group) and eyes that underwent a $2.4 \mathrm{~mm}$ temporal $\mathrm{CCl}$ (temporal $\mathrm{CCl}$ group). The mean $\mathrm{J}_{0}$ and $\mathrm{J}_{45}$ values of the total cornea were greater in the nasal $\mathrm{CCl}$ group than in the temporal $\mathrm{CCl}$ group at 2 and 4 weeks postoperatively, but did not differ significantly between groups at 2 days and at 8 weeks postoperatively. The $p$ values refer to the difference in the $J_{0}$ and $\mathrm{J}_{45}$ between groups. * Statistically significant difference between the nasal and temporal CCI groups. D, dioptre.

and other continuous variables followed a normal distribution, and therefore parametric tests were applied. The SIA and other continuous variables were compared between groups using an unpaired t-test. The $\mathrm{J}_{0}$ and $\mathrm{J}_{45}$ were compared between groups using multivariate analysis of variance. The ratio of men and women and other discrete variables were compared using the $\chi^{2}$ test or Fisher's exact test. To avoid a type I error, we modified the significance level according to the adjustment method of the false discovery rate using the Benjamini-Hochberg procedure. ${ }^{22}$ Because the primary endpoints were the magnitude of SIA, and the $\mathrm{J}_{0}$ and $\mathrm{J}_{45}$ of the total cornea, we determined that a $\mathrm{p}$ value of 0.0214 would provide an acceptable alpha level.

\section{RESULTS}

One hundred eyes of 100 patients were recruited, and all patients underwent the scheduled examinations. Because the appearances of eyes that had nasal and temporal CCIs were similar, the examiners were unaware of which eye underwent nasal or temporal CCI. Patients' characteristics and surgical outcomes did not differ significantly between groups (table 1). The mean CCI length was $1.55 \pm 0.17 \mathrm{~mm}$ in the nasal CCI group and $1.57 \pm 0.14 \mathrm{~mm}$ in the temporal CCI group; it was similar between groups $(\mathrm{p}=0.4632)$.
The mean magnitude of the SIA of the total cornea was significantly greater in the nasal CCI group than in the temporal CCI group at 2 days and 2 weeks postoperatively $(\mathrm{p} \leq 0.0214)$, and did not differ significantly between groups at 4 and 8 weeks (table 2). The percentage of eyes with SIA $\geq 1.0 \mathrm{D}$ did not differ significantly between groups at any postoperative period (online supplementary table 1). The magnitude of the SIA of the posterior cornea did not differ significantly between groups at any postoperative period.

The mean $\mathrm{J}_{0}$ and $\mathrm{J}_{45}$ values of the total cornea were significantly greater in the nasal CCI group than in the temporal CCI group at 2 and 4 weeks postoperatively ( $\mathrm{p} \leq 0.0192)$, and did not differ significantly between the groups at 2 days and 8 weeks (figure 1 ). The mean $\mathrm{J}_{0}$ and $\mathrm{J}_{45}$ values of the posterior cornea were significantly greater in the nasal CCI group than in the temporal CCI group at 2 weeks postoperatively $(\mathrm{p}=0.0007)$, and did not differ significantly between the groups at the other periods. The data of the anterior cornea are shown in online supplementary figure 1. Double-angle plot analysis showed similar results (online supplementary figure 2). When we assumed differences of $0.3 \mathrm{D}$ in both $\mathrm{J}_{0}$ and $\mathrm{J}_{45}$ to be clinically meaningful, 100 patients provided a statistical power of more than $93 \%$ for the total cornea and $100 \%$ for the posterior cornea (online supplementary table 2). 


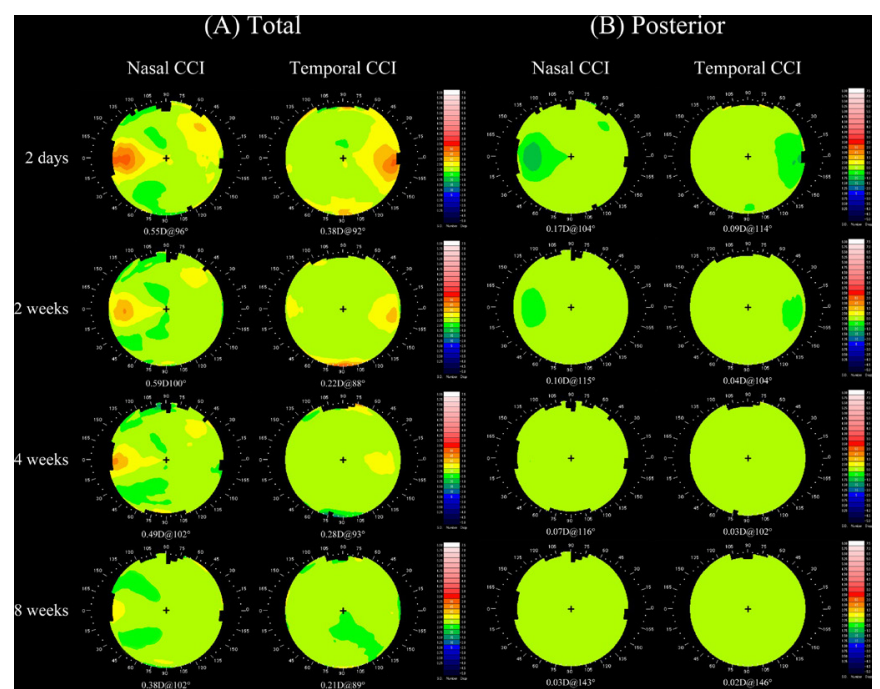

Figure 2 Mean shape changes of the total $(A)$ and posterior (B) cornea shown in the average of difference map in eyes that underwent a $2.4 \mathrm{~mm}$ nasal clear corneal incision ( $\mathrm{CCl}$; nasal $\mathrm{CCl}$ group) and eyes that underwent a $2.4 \mathrm{~mm}$ temporal $\mathrm{CCl}$ (temporal $\mathrm{CCl}$ group). (A) For the total cornea, wedge-shaped flattening and coupled steepening were observed around the CCls in the early postoperative periods in both groups, and these changes diminished over time. These changes were more prominent and extended closer to the central cornea in the nasal $\mathrm{CCl}$ group than in the temporal $\mathrm{CCl}$ group until 4 weeks. At 8 weeks postoperatively, slight coupled steepening was observed in the temporal $\mathrm{CCl}$ group, while slight peripheral flattening and coupled steepening were observed in the nasal $\mathrm{CCl}$ group; the difference between groups became slight by 8 weeks postoperatively. (B) A focal steepening occurred around both the nasal and temporal CCls at 2 days after surgery, and this steepening was slightly greater in the nasal $\mathrm{CCl}$ group than in the temporal CCl group. This wound-related steepening rapidly diminished and was not detected at 4 weeks after surgery or later in either group.

For the total cornea, in the nasal CCI group, wedge-shaped flattening and coupled steepening were observed around the CCIs at 2 days postoperatively, and these changes diminished

Table 3 Comparison of the mean $( \pm \mathrm{SD})$ irregular astigmatism components of the total cornea between eyes that underwent a 2.4 $\mathrm{mm}$ nasal clear corneal incision (CCl; nasal $\mathrm{CCl}$ group) and eyes that underwent a $2.4 \mathrm{~mm}$ temporal $\mathrm{CCl}$ (temporal $\mathrm{CCl}$ group)

\begin{tabular}{|llll}
\hline & $\begin{array}{l}\text { Nasal CCI } \\
\text { group }\end{array}$ & $\begin{array}{l}\text { Temporal CCI } \\
\text { group }\end{array}$ & P values \\
\hline $\begin{array}{l}\text { Higher order irregularity } \\
\text { component }\end{array}$ & & & \\
\hline Preoperatively & $0.15 \pm 0.04$ & $0.16 \pm 0.06$ & 0.1114 \\
\hline 2 days postoperatively & $0.31 \pm 0.10$ & $0.24 \pm 0.10$ & $0.0015^{*}$ \\
\hline 2 weeks postoperatively & $0.22 \pm 0.06$ & $0.19 \pm 0.09$ & 0.0814 \\
\hline 4 weeks postoperatively & $0.20 \pm 0.07$ & $0.17 \pm 0.05$ & $0.0202^{*}$ \\
\hline 8 weeks postoperatively & $0.18 \pm 0.06$ & $0.17 \pm 0.06$ & 0.3320 \\
\hline Asymmetry component & & & 0.2917 \\
\hline Preoperatively & $0.45 \pm 0.21$ & $0.51 \pm 0.33$ & 0.5950 \\
\hline 2 days postoperatively & $0.70 \pm 0.34$ & $0.75 \pm 0.47$ & 0.9651 \\
\hline 2 weeks postoperatively & $0.50 \pm 0.21$ & $0.50 \pm 0.25$ & 0.8280 \\
\hline 4 weeks postoperatively & $0.48 \pm 0.19$ & $0.49 \pm 0.29$ & 0.7315 \\
\hline 8 weeks postoperatively & $0.46 \pm 0.21$ & $0.48 \pm 0.27$ & \\
\hline *Statistically significant difference between groups. & & \\
\hline
\end{tabular}

over time (figure 2). In the temporal CCI group, the wedgeshaped flattening occurred around the CCIs at 2 days postoperatively, and this change diminished by 4 weeks. These changes were more prominent and extended closer to the central cornea in the nasal CCI group than in the temporal CCI group until 4 weeks. At 8 weeks postoperatively, a slight coupled steepening remained in the temporal CCI group, while slight peripheral flattening and a coupled steepening were detected in the nasal CCI group; the difference in the map between groups became small. The difference in the induced astigmatism decreased to $0.17 \mathrm{D}$ at 8 weeks. For the posterior cornea, a focal steepening occurred around both CCIs at 2 days postoperatively; this steepening was slightly greater in the nasal CCI group than in the temporal CCI group (figure 2). This steepening rapidly diminished in both groups and was not detected by 4 weeks. The maps of the anterior cornea are shown in online supplementary figure 3.

The mean higher order irregularity component of the total cornea was significantly greater in the nasal CCI group than in the temporal CCI group at 2 days and 4 weeks postoperatively $(\mathrm{p} \leq 0.0202$; table 3$)$, and did not differ significantly between the groups at 2 and 8 weeks. The mean asymmetry component did not differ significantly between the groups at any postoperative period.

The mean UDVA and CDVA (online supplementary table 3), ocular total, coma-like and spherical-like aberrations (online supplementary table 4) did not differ significantly at any follow-up period.

\section{DISCUSSION}

The videokeratographic findings revealed a wedge-shaped flattening in the total cornea and a focal steepening in the posterior cornea around the CCIs postoperatively. In the early periods, these changes were greater in the nasal CCI group than in the temporal CCI group. The wound-related changes in the total and posterior cornea, however, diminished over time in both groups. Accordingly, at 8 weeks postoperatively, the difference in the corneal shape changes became small between groups. The intergroup difference in the induced astigmatism decreased to only $0.17 \mathrm{D}$. Thus, the difference in the total and posterior corneal shape became clinically irrelevant by 2 months postoperatively in eyes with a $2.4 \mathrm{~mm}$ nasal or a temporal CCI.

The SIA was a with-the-rule shift in the total cornea and an against-the-rule shift in the posterior cornea, and the shift towards oblique astigmatism was slight. These changes in total regular astigmatism were significantly greater in the nasal CCI group than in the temporal CCI group at 2 and 4 weeks postoperatively, but did not differ significantly between groups at 8 weeks. Additionally, the higher order irregularity of the total cornea was significantly greater after nasal CCI than after temporal CCI in the early periods, but was similar at 8 weeks. These findings suggest that all changes in the total and posterior cornea became comparable between the nasal and temporal CCI groups by 8 weeks postoperatively if the incision width was $2.4 \mathrm{~mm}$ or less.

The mean ocular HOAs and visual acuity were not significantly different between groups at any postoperative period. Thus, although the surgically induced changes were different in the early periods, the differences did not markedly affect the visual outcomes.

Previous studies demonstrated that the SIA of the anterior cornea is significantly greater after nasal incision than after temporal incision when the incision width is $2.8 \mathrm{~mm}$ or more. ${ }^{5-9}$ Barequet $e t a l^{6}$ reported a significant difference in the mean SIA following a nasal CCI $(1.65 \mathrm{D})$ compared with that following a 
temporal CCI $(0.74 \mathrm{D})$ at 6 weeks postoperatively; the difference was significant. In contrast, the present study demonstrated that corneal shape and astigmatic changes of the total and posterior cornea became comparable between eyes with a 2.4 $\mathrm{mm}$ nasal CCI and those with a temporal CCI within 2 months postoperatively. This inconsistency was probably due to the smaller incision width and evaluation of the total cornea in this study. Recently, the standard CCI width became $2.4 \mathrm{~mm}$ or less. Because the outcomes did not differ at 2 months postoperatively, we consider that surgeons can choose the nasal or temporal incision according to preference.

A potential limitation of the present study is the relatively short follow-up duration. Corneal shape changes rapidly diminish and stabilise within 2 months postoperatively, however, when the incision width is $2.4 \mathrm{~mm}$ or less. ${ }^{23}$ When the incision width is $2.4 \mathrm{~mm}$ or less, a longer follow-up may not be necessary.

In conclusion, corneal shape and astigmatic changes were comparable at 2 months postoperatively between eyes that underwent a $2.4 \mathrm{~mm}$ nasal CCI and eyes that underwent a temporal CCI, although in the early periods the changes were greater after nasal CCI than after temporal CCI. Accordingly, we believe that surgeons do not need to perform the temporal incision considering the difficulties in changing their position. Whether the wound stability and frequency of anterior chamber contamination differ between these two incision sites, however, remains unclear. Further studies are needed to compare the wound stability and frequency of the anterior chamber contamination between nasal and temporal CCIs.

\section{Acknowledgements The authors thank Koji Yonemoto, PhD, for statistical} assistance.

Contributors $\mathrm{KH}$ : study conception, study design, data analysis/interpretation of data, and drafting and revising of work. TS: data acquisition and final approval. MY: data acquisition and final approval. KY: study conception, data acquisition and final approval.

Funding The authors have not declared a specific grant for this research from any funding agency in the public, commercial or not-for-profit sectors.

Competing interests None declared.

Patient consent Obtained.

Ethics approval The Institutional Review Board of Hayashi Eye Hospital granted approval for the study.

Provenance and peer review Not commissioned; externally peer reviewed.

Open access This is an open access article distributed in accordance with the Creative Commons Attribution Non Commercial (CC BY-NC 4.0) license, which permits others to distribute, remix, adapt, build upon this work non-commercially, and license their derivative works on different terms, provided the original work is properly cited, appropriate credit is given, any changes made indicated, and the use is non-commercial. See: http://creativecommons.org/licenses/by-nc/4.0/. (c) Author(s) (or their employer(s)) 2019. Re-use permitted under CC BY-NC. No commercial re-use. See rights and permissions. Published by BMJ.

\section{REFERENCES}

1 Leaming DV. Practice styles and preferences of ASCRS members-2003 survey. J Cataract Refract Surg 2004;30:892-900.

2 Elder M, Leaming D, Hoy B. New Zealand cataract and refractive surgery survey 2004. Clin Exp Ophthalmol 2006:34:401-10.

3 Yoon SC, Jung JW, Sohn HJ, et al. Cataract and refractive surgery in; a survey of KSCRS members from 1995 2006. Korean J Ophthalmol 2009;23:142-7.

4 Hayashi K, Yoshida M, Hayashi H. Postoperative corneal shape changes: microincision versus small-incision coaxial cataract surgery. J Cataract Refract Surg 2009;35:233-9.

5 Kohnen S, Neuber R, Kohnen T. Effect of temporal and nasal unsutured limbal tunnel incisions on induced astigmatism after phacoemulsification. J Cataract Refract Surg 2002;28:821-5.

6 Barequet IS, Yu E, Vitale S, et al. Astigmatism outcomes of horizontal temporal versus nasal clear corneal incision cataract surgery. J Cataract Refract Surg 2004;30:418-23.

7 Altan-Yaycioglu R, Akova YA, Akca S, et al. Effect on astigmatism of the location of clear corneal incision in phacoemulsification of cataract. J Refract Surg 2007;23:515-8.

8 Pakravan M, Nikkhah $\mathrm{H}$, Yazdani S, et al. Astigmatic outcomes of temporal versus nasal clear corneal phacoemulsification. J Ophthalmic Vis Res 2009;4:79-83.

9 Tejedor J, Pérez-Rodríguez JA. Astigmatic change induced by 2.8-mm corneal incisions for cataract surgery. Invest Ophthalmol Vis Sci 2009:50:989-94.

10 Koch DD, Ali SF, Weikert MP, et al. Contribution of posterior corneal astigmatism to total corneal astigmatism. J Cataract Refract Surg 2012;38:2080-7.

11 Nemeth G, Berta A, Lipecz A, et al. Evaluation of posterior astigmatism measured with Scheimpflug imaging. Cornea 2014;33:1214-8.

12 Koch DD, Jenkins RB, Weikert MP, et al. Correcting astigmatism with toric intraocular lenses: effect of posterior corneal astigmatism. J Cataract Refract Surg 2013:39:1803-9.

13 Ueno Y, Hiraoka T, Beheregaray S, et al. Age-related changes in anterior, posterior, and total corneal astigmatism. J Refract Surg 2014;30:192-7.

14 Cipriani V, Quartilho A, Bunce C, et al. Ophthalmic statistics note 7: multiple hypothesis testing — to adjust or not to adjust. Br J Ophthalmol 2015;99:1155-7.

15 Chylack LT, Wolfe JK, Singer DM, et al. The lens opacities classification system III. The longitudinal study of cataract study group. Arch Ophthalmol 1993;111:831-6.

16 Hayashi K, Ogawa S, Yoshida M, et al. Wound stability and surgically induced corneal astigmatism after transconjunctival single-plane sclerocorneal incision cataract surgery. Jpn J Ophthalmol 2017;61:113-23.

17 Guilbert E, Saad A, Grise-Dulac A, et al. Corneal thickness, curvature, and elevation readings in normal corneas: combined Placido-Scheimpflug system versus combined Placido-scanning-slit system. J Cataract Refract Surg 2012;38:1198-206.

18 Bao F, Savini G, Shu B, et al. Repeatability, reproducibility, and agreement of two Scheimpflug-Placido anterior corneal analyzers for posterior corneal surface measurement. J Refract Surg 2017;33:524-30.

19 Hamer CA, Buckhurst H, Purslow C, et al. Comparison of reliability and repeatability of corneal curvature assessment with six keratometers. Clin Exp Optom 2016:99:583-9.

20 Thibos LN, Horner D. Power vector analysis of the optical outcome of refractive surgery. J Cataract Refract Surg 2001;27:80-5.

21 López-Miguel A, Martínez-Almeida L, González-García MJ, et al. Precision of higherorder aberration measurements with a new Placido-disk topographer and HartmannShack wavefront sensor. J Cataract Refract Surg 2013;39:242-9.

22 Benjamini $Y$, Hochberg $Y$. Controlling the false discovery rate: a practical and powerful approach to multiple testing. J Roy Statis Soc Ser B 1995:57:298-300.

23 Hayashi K, Yoshida M, Hirata A, et al. Changes in shape and astigmatism of total, anterior, and posterior cornea after long versus short clear corneal incision cataract surgery. J Cataract Refract Surg 2018;44:39-49. 